\title{
Identification of the Model of Solid Particle Deposition in the Gravity Separator
}

\author{
Nusaba Kh. Gamzaeva* \\ Azerbaijan State Oil and Industry University \\ Baku, Azerbaijan
}

Received 26.08.2019, received in revised form 21.12.2019, accepted 19.01.2020

\begin{abstract}
The unsteady process of deposition of a solid particle in a stationary liquid in a gravity separator is considered. To describe this process, a mathematical model is proposed that takes into account the gravity of the particle, the buoyant force of Archimedes and the resistance force of the liquid described by the quadratic Newton's law of resistance. Within the framework of the proposed model, the problem of identifying the resistance coefficient according to an additional given condition regarding the position of the particle in the separator at a fixed time is posed. To solve the problem, the analytical solution of the model is first determined, and then the resulting solution is substituted in an additional condition specified in the integral form. After integration, the identification problem is reduced to a nonlinear transcendental equation with respect to the desired resistance coefficient. The method of simple iteration is used for the numerical solution of the obtained nonlinear equation. Based on the proposed computational algorithm, numerical experiments were carried out for the model data.
\end{abstract}

Keywords: gravity separator, deposition, liquid resistance force, resistance coefficient, identification problem.

Citation: Gamzaeva N.Kh. Identification of the model of solid particle deposition in the gravity separator, J. Sib. Fed. Univ. Chem., 2020, 13(1), 46-52. DOI: 10.17516/1998-2836-0156

(C) Siberian Federal University. All rights reserved

This work is licensed under a Creative Commons Attribution-NonCommercial 4.0 International License (CC BY-NC 4.0).

* Corresponding author E-mail address: hemzeyevanusaba90@mail.ru 


\title{
Идентификация модели осаждения твердой частицы \\ в гравитационном сепараторе
}

\author{
H.X. Гамзаева \\ Азербайджанский государственный \\ университет нефти и промышленности \\ Азербайджан, Баку
}

\begin{abstract}
Аннотаиия. Рассматривается нестационарный процесс осаждения твердой частицы в покоящейся жидкости в гравитационном сепараторе. Для описания данного процесса предлагается математическая модель, учитывающая действия силы тяжести частицы, выталкивающей силы Архимеда и силы сопротивления жидкости, описываемой квадратичным законом сопротивления Ньютона. В рамках предложенной модели поставлена задача идентификации коэффициента сопротивления по дополнительно заданному условию относительно положения частицы в сепараторе в некоторый фиксированный момент времени. Для решения поставленной задачи сначала определяется аналитическое решение модели, а затем полученное решение подставляется в дополнительное условие, заданное в интегральном виде. После интегрирования задача идентификации сводится к нелинейному трансцендентному уравнению относительно искомого коэффициента сопротивления. Для численного решения полученного нелинейного уравнения используется метод простой итерации. На основе предложенного вычислительного алгоритма были проведены численные эксперименты для модельных данных.
\end{abstract}

Ключевые слова: гравитационный сепаратор, осаждение, сила сопротивления жидкости, коэффициент сопротивления, задача идентификации.

Цитирование: Гамзаева, Н.Х. Идентификация модели осаждения твердой частицы в гравитационном сепараторе / H.Х. Гамзаева // Журн. Сиб. федер. ун-та. Химия, 2020. 13(1). С. 46-52. DOI: 10.17516/1998-2836-0156

\section{Введение}

В настоящее время применение современных технологий в нефтедобыче существенно повышает концентрацию твердых частиц из песка, глины, известняка и других пород в добываемой нефти. Различные твердые частицы, находящиеся в нефти, повреждают нефтепроводы и оборудование при перекачке нефти, образуют отложения в теплообменных аппаратах и других емкостях, усложняют процесс переработки нефти и т.д. Частицы также содействуют образованию стойких эмульсий нефти с пластовой водой. В практике для отделения частиц породы от нефти применяют физические методы, которые включают очистку под воздействием гравитационных, центробежных, электродинамических сил, очистку путем фильтрования через пористые перегородки, а также очистку с помощью комбинации этих методов [1,2]. В нефтяной промышленности для очистки нефти от твердых частиц чаще всего применяют гравитационные сепараторы. В гравитационных сепараторах разделение твердых частиц происходит за счет гравитации, то есть частицы с меньшим удельным весом поднимаются вверх, а тяжелые

$$
-47-
$$


оседают на дно. Следует отметить, что для обеспечения эффективной работы гравитационных сепараторов необходимо иметь адекватную математическую модель процесса осаждения твердых частиц в сепараторах, на основании которой возможно установление основных закономерностей функционирования этого оборудования. Различные аспекты проблемы математического моделирования процессов движения твердых частиц в жидкостях исследованы многими авторами [3-11]. В данной работе рассматривается задача идентификации одной модели процесса осаждения твердой частицы в вертикальном гравитационном сепараторе.

\section{Постановка задачи и метод решения}

Проанализируем процесс осаждения твердой частицы в покоящейся жидкости в вертикальном гравитационном сепараторе. За начало отсчета примем точку начала движения частицы в жидкости и координатную ось $O z$ направим вертикально вниз. Предположим, что на частицу действует сила тяжести частицы $P$, направленная вертикально вниз,

$$
P=m_{p} g
$$

выталкивающая сила Архимеда $F_{A}$, направленная вертикально вверх,

$$
F_{A}=-m_{p} g \frac{\rho_{f}}{\rho_{p}}
$$

и сила сопротивления жидкости $F_{s}$, описываемая квадратичным законом сопротивления Ньютона, зависящая как от свойств жидкости, так и от скорости и формы частицы [2-11], также направленная вертикально вверх,

$$
F_{S}=-\frac{C}{2} s_{p} \rho_{f} u^{2}(t)
$$

где $u(t)$ - скорость движения частицы в направлении оси $O z$ (скорость осаждения частицы); $t$ - время; $g$ - ускорение свободного падения; $m_{p}$ - масса частицы; $\rho_{p}-$ плотность частицы; $\rho_{f}-$ плотность покоящейся жидкости в сепараторе; $s_{p}$ - максимальная площадь сечения частицы в плоскости, перпендикулярной направлению ее движения; $C$ - безразмерный коэффициент сопротивления, зависящий от формы частицы и режима движения.

Тогда согласно второму закону Ньютона математическую модель движения частицы в вертикальном гравитационном сепараторе можно представить в виде

$$
m_{p} \frac{d u(t)}{d t}=m_{p} g-m_{p} g \frac{\rho_{f}}{\rho_{p}}-\frac{C}{2} s_{p} \rho_{f} u^{2}(t)
$$

или

$$
\frac{d u(t)}{d t}=\gamma-C \lambda u^{2}(t)
$$

где $\gamma=\frac{g\left(\rho_{p}-\rho_{f}\right)}{\rho_{p}}, \lambda=\frac{s_{p} \rho_{f}}{2 m_{p}}$. При этом предполагается, что $\rho_{p}>\rho_{f}$. Предположим, что для уравнения (1) задается следующее начальное условие:

$$
-48-
$$




$$
u(0)=0 .
$$

Очевидно, что при задании параметров $m_{p}, C, \rho_{p}, \rho_{f}, s_{p}$, решив уравнение (1) с учетом начального условия (2), можно найти зависимость скорости осаждения частицы от времени. Однако необходимо отметить очень важное обстоятельство относительно коэффициента сопротивления $C$. Принято считать, что коэффициент сопротивления зависит от значения критерия Рейнольдса Re [2-6]. Для того чтобы выбрать значение коэффициента сопротивления, необходимо предварительно знать значение критерия Рейнольдса. Однако ввиду того, что в формулу для расчета критерия Рейнольдса входит неизвестная функция - скорость осаждения частицы $u(t)$, вычислить значение критерия Рейнольдса и, следовательно, коэффициент сопротивления не представляется возможным. В практических задачах для разрешения этой проблемы применяется недостаточно обоснованный и трудоемкий метод последовательных приближений. На первой стадии задается, например, ламинарный режим осаждения, а затем, определив скорость осаждения, проверяют, лежит ли критерий Рейнольдса в области, соответствующей выбранному условию. При несовпадении переходят ко второй стадии расчета и т.д. Очевидно, что при нестационарном движении твердой частицы в жидкости происходят изменения скорости и значения критерия Рейнольдса во времени. Следовательно, применение указанного подхода для исследования нестационарного движения частицы в жидкости неневозможно. В связи с этим возникает необходимость в решении задачи идентификации для модели (1), связанной с определением коэффициента сопротивления $C$.

Предположим, что коэффициент сопротивления $C$ в уравнении (1) неизвестен и подлежит определению наряду с функцией $u(t)$. Взамен этого положение частицы в некоторый фиксированный момент времени считается заданным

$$
z_{T}=\int_{0}^{T} u(t) d t
$$

где $z_{T}$ - положение частицы в момент времени $t=T$.

Таким образом, задача идентификации заключается в определении функции $u(t)$ и коэффициента $C$, удовлетворяющих уравнению (1) и условиям (2), (3).

Для решения поставленной задачи идентификации (1)-(3) сначала найдем аналитическое решение уравнения (1), удовлетворяющее начальному условию (2). Уравнение (1) является дифференциальным уравнением с разделяющимися переменными, и его решение с учетом условия (2) записывается в виде

$$
u(t)=\frac{1-\ell^{-2 \sqrt{C \lambda \gamma t}}}{1+\ell^{-2 \sqrt{C \lambda \gamma t}}} \sqrt{\frac{\gamma}{C \lambda}} .
$$

Решив уравнение (1), представленное в форме (4), подставив в уравнение (3) и выполнив интегрирование, получим следующее нелинейное трансцендентное уравнение относительно коэффициента сопротивления $C$

$$
z_{T}=\sqrt{\frac{\gamma}{C \lambda}} T+\frac{1}{C \lambda} \ln \frac{1+\ell^{-2 \sqrt{C \lambda \gamma} T}}{2} .
$$


Ввиду нелинейности уравнения (5) найти его аналитическое решение не представляется возможным. Для численного решения уравнения (5) можно использовать метод простой итерации [12].

Таким образом, определив координаты частицы $z_{T}$ в некоторый фиксированный момент времени $t=T$ путем непосредственного измерения в сепараторе, можно найти значение коэффициента сопротивления $C$ из решения уравнения (5).

\section{Результаты численных расчетов}

На основе предложенного вычислительного алгоритма были проведены численные расчеты для процесса осаждения шарообразной частицы песка с плотностью $\rho_{p}=1600$ кг/м $\mathrm{m}^{3}$ и радиусом $R=1 \mathrm{мм}=1 \cdot 10^{-3}$ м в тяжелой нефти с плотностью $\rho_{f}=980$ кг $/ \mathrm{M}^{3}$. Значения параметров $m_{p}$ и $s_{p}$ определены по формулам $m_{p}=4 \pi R^{3} \rho_{p} / 3, s_{p}=4 \pi R^{2}$. А в качестве значений экспериментально измеряемых величин $z_{T}$ и $T$ использованы модельные данные.

Результаты численных расчетов по определению коэффициента сопротивления $C$ представлены в таблице. Численные расчеты показывают, что, зафиксировав положение частицы в определенный момент времени, можно по предложенному алгоритму однозначно найти значение коэффициента сопротивления. Ввиду того, что модельные данные о положении частицы

Таблица. Результаты численного эксперимента

Table. Results of a numerical experiment

\begin{tabular}{c|c|c|c|c|c}
\hline \multirow{2}{*}{$z_{T}, \mathrm{M}$} & \multicolumn{5}{|c}{ Вычисленное значение C } \\
\cline { 2 - 6 } & $\mathrm{T}=3 \mathrm{c}$. & $\mathrm{T}=5 \mathrm{c}$. & $\mathrm{T}=8 \mathrm{c}$. & $\mathrm{T}=10 \mathrm{c}$. & $\mathrm{T}=15 \mathrm{c.}$ \\
\hline 1.0 & 0.1427 & 0.4073 & 1.0521 & 0.6473 & 3.7140 \\
\hline 1.5 & 0.0620 & 0.1797 & 0.4662 & 0.7308 & 1.6493 \\
\hline 2.0 & 0.0341 & 0.1003 & 0.2615 & 0.4103 & 0.9270 \\
\hline 2.5 & 0.0213 & 0.0637 & 0.1669 & 0.2621 & 0.5928 \\
\hline 3.0 & 0.0145 & 0.0439 & 0.1155 & 0.1817 & 0.4113 \\
\hline 3.5 & 0.0104 & 0.0320 & 0.0846 & 0.1332 & 0.3019 \\
\hline 4.0 & 0.0077 & 0.0243 & 0.0646 & 0.1018 & 0.2310 \\
\hline 4.5 & 0.0059 & 0.0190 & 0.0509 & 0.0803 & 0.1824 \\
\hline 5.0 & 0.0047 & 0.0153 & 0.0411 & 0.0649 & 0.1476 \\
\hline 5.5 & 0.0037 & 0.0135 & 0.0339 & 0.0536 & 0.1219 \\
\hline 6.0 & 0.0030 & 0.0105 & 0.0284 & 0.0449 & 0.1023 \\
\hline 6.5 & 0.0025 & 0.0088 & 0.0241 & 0.0382 & 0.0871 \\
\hline 7.0 & 0.0021 & 0.0075 & 0.0207 & 0.0329 & 0.0751 \\
\hline 7.5 & 0.0017 & 0.0065 & 0.0180 & 0.0286 & 0.0653 \\
\hline 8.0 & 0.0015 & 0.0057 & 0.0158 & 0.0251 & 0.0574 \\
\hline 8.5 & 0.0012 & 0.0050 & 0.0139 & 0.0222 & 0.0508 \\
\hline 9.0 & 0.0000 & 0.0044 & 0.0124 & 0.0197 & 0.0453 \\
\hline 10.0 & 0.0000 & 0.0039 & 0.0111 & 0.0177 & 0.0406 \\
\hline
\end{tabular}


задавали произвольно, некоторые результаты не соответствуют реальному физическому процессу. Например, для случая $T=3$ с и $z_{T}=9 ; 9.5 ; 10$ м значения коэффициента сопротивления равны нулю. Этот результат показывает, что частица через 3 с могла оказаться в положении $z_{T}=9$ м только в том случае, если коэффициент сопротивления равен нулю. Или же частица через 15 с могла оказаться в положении $z_{T}=1$ м только в том случае, если значение коэффициента сопротивления было достаточно большим $(C=3.714)$.

Анализ полученных результатов свидетельствует, что предложенный вычислительный алгоритм можно использовать при исследовании процессов осаждения твердых частиц в гравитационных сепараторах.

\section{Выводы}

В работе предложен вычислительный алгоритм для идентификации коэффициента сопротивления в модели процесса осаждения твердой частицы в гравитационном сепараторе, основанный на использовании информации о положении частицы в некоторый фиксированный момент времени. В отличие от традиционного подхода при этом не возникает необходимости в определении режима движения частицы в жидкости и использовании приближенных формул для определения коэффициента сопротивления.

\section{Список литературы / References}

1. Плановский А.Н., Николаев П.И. Процессы и аппараты химической и нефтехимической технологии. М.: Химия, 1987. 496 с. [Planovskiy A.N., Nikolaev P.I. Processes and devices of chemical and petrochemical technology. M.: Khimiya, 1987. 496 p. (In Russ.)]

2. Архипов В.А., Усанина А.С. Движение частиц дисперсной фазы в несущей среде. Томск: Издат. Дом Томского государственного университета, 2014, 252 c. [Arkhipov V.A., Usanina A.C The movement of particles of the dispersed phase in the carrier medium. Tomsk: Izdatelskiy Dom Tomskogo gosudarstvennogo universiteta, 2014. 252 p. (In Russ.)]

3. Bürger R. \& Wendland W.L. Sedimentation and suspension flows: Historical perspective and some recent developments. Journal of Engineering Mathematics 2001. Vol. 41(2), P. 101116.

4. Dorgan A.J. and Loth E. Efficient calculation of the history force at finite Reynolds numbers. Int. J. Multiphase Flow 2007. Vol. 33(8), P. 833-848.

5. Vodop'yanov I.S., Petrov A.G., Shunderyuk M.M. Unsteady sedimentation of a spherical solid particle in a viscous fluid. Fluid Dynamics 2010. Vol. 45(2), P. 254-263.

6. Zaidi A., Tsuji T., Tanaka T. A New Relation of Drag Force for High Stokes Number Monodisperse Spheres by Direct Numerical Simulation. Advanced Powder Technology 2014. Vol. 25(6), P. 1860-1871.

7. Sayed M. Derakhshania, Dingena L. Schott, Gabriel Lodewijks. Modeling particle sedimentation in viscous fluids with a coupled immersed boundary method and discrete element method. Particuology 2017. Vol. 31, P. 191-199.

8. Nouria R., Ganjia D.D., Hatami M. Unsteady sedimentation analysis of spherical particles in Newtonian fluid media using analytical methods. Propulsion and Power Research 2014. Vol. 3(2), P. 96-105. 
9. Walter F., Francesco P., Luca B. Sedimentation of finite-size spheres in quiescent and turbulent environments. J. Fluid Mekh 2016. Vol. 788, P. 640-669.

10. Mehdi F., Alireza R., Hesam M., Iman R., Davood D., Mostafa V. Analytical study of unsteady sedimentation analysis of spherical particle in newtonian fluid media. Thermal science 2018. Vol. 22(2), P. 847-855.

11. Arkhipov V.A., Usanina A.S. Regimes of Sedimentation of a Consolidated System of Solid Spherical Particles. Fluid Dynamics 2017. Vol. 52(5), P. 666-677.

12. Самарский А.А., Гулин А.В. Численные методы. М.: Наука, 1989. 432 с. [Samarskiy A.A., Gulin A.V. Numerical methods. M.: Nauka, 1989. 432 p. (In Russ.)] 